\title{
CLINICAL AND RADIOLOGICAL FEATURES OF MEDICATION-RELATED OSTEONECROSIS OF THE JAWS: COMPARISON OF STAGING SYSTEMS
}

Alessandro Antonelli ${ }^{1}$, Francesco Bennardo ${ }^{1}$, Selene Barone, Davide Caruso ${ }^{1}$, Maria Giulia Cristofaro ${ }^{1}$, Amerigo Giudice 1 Universita' degli Studi "Magna Græcia" di Catanzaro

Funding: The author(s) received no specific funding for this work.

Potential competing interests: The author(s) declared that no potential competing interests exist.

\section{Abstract}

Medication-related osteonecrosis of the jaw (MRONJ) has been recognized as one of the most disabling comorbidities associated to the assumption of bisphosphonates (BPs) and antiresorptive drugs.

To date, several classifications identify the stages of this pathology to propose different protocols for a better management way.

This pilot retrospective study aims to compare two of the most used MRONJ classifications (AAOMS vs SIPMO-SICMF), analyzing CT scans and clinical features of patients with documented MRONJ.

For each patient, clinical features and radiological findings were analyzed before any therapeutic procedure. The diagnostic differences between the AAOMS and SIPMO-SICMF classification were recorded and analyzed according to the treatment outcomes. These findings highlight different approaches for the patient's treatment related to the MRONJ stages.

Our results suggest a continuous update of the international guidelines to uniform the treatment for the improvement of the patients' quality of life.

\section{Background}

Medication-related osteonecrosis of the jaw (MRONJ) is a rare complication of bone modifying agents (BMA) used in the

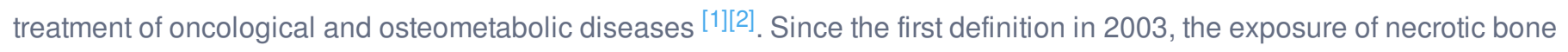
in the oral cavity has been considered the significant clinical sign allowing a diagnosis of MRONJ ${ }^{[3]}$.

The AAOMS position paper is the most used staging system to evaluate the condition of MRONJ patients ${ }^{[4]}$ however, several authors suggested an alternative MRONJ classification system including clinical and radiological signs to improve diagnostic criteria and treatment strategies ${ }^{[5]}$.

This retrospective analysis aimed to compare two MRONJ classification systems (AAOMS vs SIPMO-SICMF), analyzing CT scans and clinical features of patients with documented MRONJ.

\section{Materials and Methods}


This study was designed as single-center retrospective study enrolling patients with a clinical diagnosis of MRONJ. Anamnestic data and clinical features were recorded and specific radiological exams (orthopantomography or cone-beam computed tomography) were examined for a better identification of radiological signs. Exclusion criteria were: previous history of radiotheraphy int the head and neck region, metastatic bone disease of the maxillofacial area, incomplete radiological exams.

Two expert physician analyzed signs, symptoms and radiological findings; one clinician assigned the MRONJs' stage to each patient in accordance with the AAOMS staging, the other according to the SIPMO/SICMF staging.

\section{$\underline{\text { Results }}$}

A total of 33 patients ( 21 female and 12 male, mean age $78.9 \pm 6.2$ years) were enrolled in this study. Fifteen patients were affected by oncological pathologies and 18 received BMA for osteometabolic diseases. According to drug administration, 15 patients were treated with intravenous drug therapy and 18 patients received an oral pharmacological treatment. A total of 15 maxilla and 21 mandibular lesions were evaluated. In 9 patients $(27,2 \%)$ necrotic bone exposure in the oral cavity was not recorded, while in 24 (72,7\%) patients the necrotic bone exposure was detected. The radiological evaluation images allowed to assess that the signs mostly encountered in our sample were the periosteal involvement (90\%) and the localized osteosclerosis $(81,8 \%)$. According to SIPMO/SICMF classification, the following results were recorded: 6 patients with stage $1 \mathrm{~B}(18 \%), 18$ patients with stage $2 \mathrm{~B}(54 \%)$, and 9 patients with stage $3(27 \%)$. In accordance with the AAOMS classification, 9 patients with stage $0(27 \%)$, 3 patients with stage $1(9 \%)$, 3 patients with stage $2(9 \%)$, and 18 patients with stage $3(54 \%)$ were identified.

\section{Conclusions}

Although the results of this study are based on a small sample, they highlighted a heterogeneity in MRONJ patients staging with AAOMS and SIPMO-SICMF classifications. To date, an unified classification based on a detailed analysis of clinical and radiological signs could be desirable to improve the evaluation of this disease and standardize the therapeutic approach for MRONJ patients.

\section{References}

1. 'Morten Schiodt, Sven Otto, Stefano Fedele, Alberto Bedogni, et al. (2019). Workshop of European task force on medication-related osteonecrosis of the jaw-Current challenges. Oral Dis, vol. 25 (7), 1815-1821. doi:10.1111/odi.13160.

2. ^Amerigo Giudice, Selene Barone, Federica Diodati, Alessandro Antonelli, et al. (2020). Can Surgical Management Improve Resolution of Medication-Related Osteonecrosis of the Jaw at Early Stages? A Prospective Cohort Study. Journal of Oral and Maxillofacial Surgery, vol. 78 (11), 1986-1999. doi:10.1016/j.joms.2020.05.037.

3. ^Robert E Marx. (2003). Pamidronate (Aredia) and zoledronate (Zometa) induced avascular necrosis of the jaws: a growing epidemic. Journal of Oral and Maxillofacial Surgery, vol. 61 (9), 1115-1117. doi:10.1016/s02782391(03)00720-1.

4. 'Salvatore L. Ruggiero, Thomas B. Dodson, John Fantasia, Reginald Goodday, et al. (2014). American Association of 
Oral and Maxillofacial Surgeons Position Paper on Medication-Related Osteonecrosis of the Jaw-2014 Update. Journal of Oral and Maxillofacial Surgery, vol. 72 (10), 1938-1956. doi:10.1016/j.joms.2014.04.031.

5. `A Bedogni, V Fusco, A Agrillo, G Campisi. (2012). Learning from experience. Proposal of a refined definition and staging system for bisphosphonate-related osteonecrosis of the jaw (BRONJ). Oral Diseases, vol. 18 (6), 621-623. doi:10.1111/j.1601-0825.2012.01903.x. 